\title{
Rheological properties of sweet potato starch-date syrup gel
}

\author{
Abdellatif Abdelhakim MOHAMED ${ }^{1 *}$ (D), Mohammed Salih ALAMRI ${ }^{1}$, Shahzad HUSSAIN ${ }^{1}$, \\ Mohamed AbduRabu IBRAHEEM ${ }^{1}$, Akram Abdo QASEM ${ }^{1}$
}

\begin{abstract}
Date syrup (DS), sugars (SG) and sweet potato starch (SPS) composites were investigated using dynamic rheometry, rapid visco-amylograph (RVA), and texture analysis (TPA). HPLC analysis revealed that DS sugar composition was $55 \%$ glucose and $45 \%$ fructose. Starch gels ( $0 \%$ to $50 \%$ DS or SG) were precooked in RVA. SPS starch gel is considered elastic because the elastic modulus $\left(G^{\prime}\right)$ was much greater than the viscous modulus $\left(G^{\prime \prime}\right)$. The comparison between the effect of DS versus SG revealed differences on the peak viscosity, setback, $G^{\prime}$ and $G^{\prime \prime}$, complex viscosity $\left(\eta^{*}\right)$, $\tan \delta$, and gel hardness. The decline in complex viscosity $\left(\eta^{*}\right)$ indicates shear thinning behavior. Despite the similarity in sugar content between DS and SG, as shown by the HPLC analysis, the data presented here disclosed how DS had more impact on the pasting and rheological properties of sweet potato starch compared to SG.
\end{abstract}

Keywords: starch; rheology; date syrup; pasting; sweet potato.

Practical Applications: 1. Despite the similar sugar content between date syrup (naturally found) and prepared sugar solution, sweet potato dynamic rheological, textural and pasting properties were found to be different; 2 . The comparison between the effect of DS versus SG revealed differences on the peak viscosity, setback, $G^{\prime}$ and $G^{\prime \prime}$, as well as gel hardness.

\section{Introduction}

Sweet potato is a native of Africa used mostly in baked products, noodles and different sauces and is a major source of starch. It has granules size around 3-40 $\mu \mathrm{m}, 17-30 \%$ amylose content, gelatinization temperature between 60 and $70^{\circ} \mathrm{C}$, and a mixed x-ray diffraction pattern of A- and C-type (Noda et al., 1998). Compared to corn starch, it exhibits higher pasting viscosity and paste clarity. Even though the effect of date syrup and different sugars on the pasting properties of some starches has been explored, a number of additives, such as gums, were reported to modify the physicochemical properties of starch. Xanthan gum was reported to increase the G' (elasticity), G'(viscous property), and $\eta^{\star}$ (complex viscosity) as a function of gum level, while the $\tan \delta$ values decreased signifying a more elastic and less viscous paste. The addition of date syrup was reported to increase the pasting temperature, and $\mathrm{G}^{\prime}$, with possible reduction on starch retrogradation (Mohamed \& Babucurr, 2015). Date syrup was found to decrease the peak viscosity of low amylose rice starch and increases the final viscosity and the setback, whereas the G' was unchanged (Mohamed \& Babucurr, 2017).

One of the main reasons for adding sugars or other sweeteners to starchy products is to develop required taste. According to thermal analysis, sugars were proven to increase gelatinization temperature and influence starch pasting properties (Ahmad \& Williams, 1999; Baek et al., 2004; Chang et al., 2014; Gunaratne et al., 2007; Maaruf et al., 2001; Sopade et al., 2004; Zhang et al., 2013). Nevertheless, inconsistent reports on the effect of sugars on starch properties are present in the literature (Pongsawatmanit et al., 2007; Chang et al., 2014). Zhang et al.,
(2013) reported that sugars increased starch gelatinization activation energy as a function of increased level of sugar, but reduced the rate constant resulting in delayed starch gelatinization temperature. They also reported that sucrose was more effective than glucose. Kim (1992) reported significant increase in the DSC peak temperature of wheat starch but not on the $\Delta \mathrm{H}$. Sucrose esters were reported to increase starch gelatinization temperature causing sponge cakes to collapse right after removal from the oven (Kim, 1992). Inadequate starch gelatinization was reported to be the reason for the collapse. Sugars can be ranked as: $x y l o s e>$ sucrose $>$ glucose $>$ control with respect to influence on $G^{\prime}$ of starch gel (Chang et al., 2014).

In the course of the swelling step of starch gelatinization, sugars can penetrate into the granule and complex with amylose and amylopectin eliciting higher gelatinization temperature (Chiotelli et al., 2000). Diffractograms of starch and starch-sugar systems showed how sugars cause disorder inside the amorphous region of the starch granule causing increase in the gelatinization temperature (Sikora \& Pielichowski, 1999). The decrease in starch gel hardness parameters and increase in springiness parameters indicated that low molecular weight sugars could retard tapioca starch retrogradation. FTIR and TPA analysis showed reliable results of the effect of sugars on starch retrogradation which is in line with those of DSC and retrogradation kinetics analysis.

Miles et al. (1985) attributed the elastic ( $\left.G^{\prime}\right)$ response of starch gel to the physical entanglement of neighbouring molecules or to the intermolecular association of amylose chains released 
from the swollen granules. The several magnitudes increase in $\mathrm{G}^{\prime}$ of pure starch gel (no sugar) can reach its maximum at $4^{\circ} \mathrm{C}$. Nonetheless, corn starch-sugar system exhibited no significant variations in the temperature, but a drop on $G^{\prime}$ values were detected at high concentrations of sugars. This signifies the cause of the large decrease in the rate of change in amylose conformation during cooling caused by sugars (Evageliou et al., 2000). The use of nanotechnology can be used to help in developing products with desired properties, such as less water separation through less re-association of amylose and amylopectin (Ma'abreh et al., 2018).

Date is a fruit of date palms trees (Phoenix dactylifera) cultivated in many parts of the world especially in the Middle East. Date syrup is a product of date fruits which can be produced in several concentrations by extraction and condensation. Glucose and fructose are the major sugars present in date syrup. Date syrup tastes sweeter than sucrose and has a unique good flavor (Al-Hooti et al., 2002). Date syrup contains, in addition to sugar, macro and micro elements which may play important role of considering date syrup as rich nourishment. In addition to the high sugar content, date syrup is considered a good source of minerals such as potassium, magnesium and calcium (Al-Khateeb, 2008).

The effect of monosaccharides (glucose and fructose) as well as sucrose on the functional properties of starch has been reported in the literature. However, a comparison study between the effect of monosaccharides and high Brix date syrup on sweet potato starch gelatinization has not been done to the best of our knowledge. The objective of this work is to determine and compare the effect of date syrup and sugars on the rheological and pasting properties of sweet potato and corn starch gel. In this work concentrations of a mixture of glucose/fructose were selected to mimic sugars concentrations found naturally in date syrup so that a meaningful comparison can be made. The novelty of this work is based on replacing processed sugars with a natural source which can potentially find application in the food industry such as the baking, beverage, and snack industry.

\section{Material and methods}

\subsection{Materials}

Fresh date syrup (DS) 75 Brix was supplied by Esten date \&fresh date Est, a local producer. Sweet potato starch was isolated from fresh sweet potato tubers purchased from a local market. Analytical grade glucose and fructose were purchased from Wells Lawrence House (126 Back Church Lane, London, EI IFH, UK).

\subsection{Sweet potato starch isolation}

Five $\mathrm{Kg}$ of sweet potato (SPS) tuber ((Ipomoea batatas) was thoroughly washed, peeled, diced, and blended in distilled water (50/50) for 3 min using kitchen aid blender (kitchen aid, Benton Harbor, MI, United States). The slurry was then filtered through a muslin cloth and the residue was re-suspended and reprocessed same way. The supernatant from the first and the second filtration was combined sieved through 200 mesh sieve and the liquid leaked through the sieve was allowed to settle for $1 \mathrm{~h}$ at room temperature $\left(23^{\circ} \mathrm{C}\right)$. The settled material was re-suspended in distilled water and centrifuged at $2000 \mathrm{x} \mathrm{g}$ for 15 minutes and the top dark layer was removed. The white material at the bottom of the bottle was re-suspended in distilled water and centrifuged up until pure white starch was obtained. The isolated starch was air-dried at room temperature and stored in air -tight jars below $5^{\circ} \mathrm{C}$.

\subsection{High Pressure Liquid Chromatographic analysis (HPLC)}

Glucose and fructose content of the date syrup was determined according to AOAC standard method 16.12 (Association of Official Analytical Chemists, 1990). Shimadzu HPLC system (LC-10A HPLC Series, Shimadzu, Kyoto, Japan) equipped with a refractive index detector (RID-10A) and a UV/Vis detector (SPD-20A) monitored at $210 \mathrm{~nm}$. Sugars analysis was done using Aminex HPX-87H column $(300 \times 7.8 \mathrm{~mm})($ Bio-Rad $)$ at $55^{\circ} \mathrm{C}$ at $0.3 \mathrm{~mL} \mathrm{~min}^{-1}$ flow. Isocratic solvent mixture of $0.045 \mathrm{~N} \mathrm{H}_{2} \mathrm{SO}_{4}$ with $6 \%$ acetonitrile solvent $(\mathrm{v} / \mathrm{v})$ was designated as a mobile phase. An aliquot of date syrup diluted with $100 \mathrm{~mL}$ of distilled water, filtered through $0.45 \mathrm{~mm}$ membrane and $20 \mu \mathrm{L}$ was injected at a total running time of $10 \mathrm{~min}$.

\subsection{Blends preparation}

Blends were prepared in two ways. A replacement or addition, where portion of the starch was replaced with DS or SG or added to a fixed amount of starch. Sugar solutions were prepared at a ratio of 55:45 glucose to fructose according to their representation in the date syrup determined by the HPLC analysis. The total amount of starch used was $2.8 \mathrm{~g}$ for the addition study and varying amounts of starch for the replacement experiment. Blends were prepared at $0,5,15,20,30$, and 50\% date syrup (DS) of the starch weight (14\% moisture content). For example, in the replacement experiment the $5 \%$ DS or SG is equal to $0.14 \mathrm{~g}$ solids of DS or SG in the total weight of $2.8 \mathrm{~g}$ and the remaining $2.66 \mathrm{~g}$ is starch. Since DS is $75 \%$ solids, $0.14 \mathrm{~g}$ solids are in $0.187 \mathrm{~g}$ of DS. That means for the $5 \%$ DS blend, $2.66 \mathrm{~g}$ of starch $+0.187 \mathrm{~g}$ of DS. The remaining blends were calculated the same way.

\subsection{Rapid Visco-Analyzer measurements (RVA)}

Rapid Visco-analyzer (Newport Scientific, Sydney, Australia) was used to determine the pasting properties of the sweet potato starch / date syrup or sugars gels. A total weight of $2.8 \mathrm{~g}$ at $14 \%$ moisture basis was weighed into aluminum RVA canister and the total weight was adjusted to $28 \mathrm{~g}$ by adding distilled water. The obtained slurry was tested according to Alamri et al. (2016). A steady increase in temperature to $95^{\circ} \mathrm{C}$ at $10.3^{\circ} \mathrm{C} / \mathrm{min}$, a holding step at $95^{\circ} \mathrm{C}$ for $4 \mathrm{~min}$, a linear temperature decrease to $50{ }^{\circ} \mathrm{C}$ at $22,5^{\circ} \mathrm{C} / \mathrm{min}$, and a final isothermal step at $50{ }^{\circ} \mathrm{C}$ for $2 \mathrm{~min}$. The agitation was $960 \mathrm{rpm}$ for the first $10 \mathrm{sec}$ and then $160 \mathrm{rpm}$. All measurements were done in 2 replicates. Thermocline software provided by the RVA manufacture was used to process the data.

\subsection{Dynamic rheometry}

Dynamic rheology measurements were carried out on starch paste pre-cooked in RVA experiments using DHR- Hybrid Rheometer (TA instruments, DE, USA). Prior to the shear 
measurements, a strain-sweep was conducted to determine the linear viscoelastic region. Small amplitude oscillatory shear tests were conducted over a frequency sweep range of $0.1-100(\mathrm{rad} / \mathrm{s})$ at $2 \%$ strain, yielding shear storage modulus $\left(\mathrm{G}^{\prime}\right)$, loss modulus $\left(\mathrm{G}^{\prime \prime}\right)$ and complex viscosity ( $\eta^{*}$ Pa.s). The frequency range used here is typically used for the frequency sweep so that $\mathrm{G}^{\prime}$, $G^{\prime \prime}$, and $\eta^{\star}$ were within the linear region. The storage modulus represents the non-dissipative element of mechanical properties, whereas the loss modulus symbolizes the dissipative component of mechanical properties of the material. Elastic behavior indicates that $G^{\prime}$ is independent of frequency and greater than $G^{\prime \prime}$. Materials can be described as solid with perfect elasticity when the phase shift angle $(\delta)$ is zero, liquid with impeccable viscosity when $\delta=90$, or somewhere in the middle. The phase angle is defined as $\delta=\tan ^{-1}\left(\mathrm{G}^{\prime \prime} / \mathrm{G}^{\prime}\right)$.

\subsection{Gel texture}

Gels prepared in RVA were transferred into $25 \mathrm{~mL}$ beakers (35 $\mathrm{mm}$ in height) with internal diameters of $30 \mathrm{~mm}$ and kept overnight at room temperature. Texture expressed as gel strength was obtained by compressing gels using Brookfield CT3 Texture Analyzer (Brookfield Engineering Laboratories, Inc. Middleboro, USA). The test was performed, after overnight storage at room temperature, in two penetration cycles at a speed of $0.5 \mathrm{~mm} / \mathrm{s}$ to a distance of $10 \mathrm{~mm}$ using $12.7 \mathrm{~mm}$ wide and $35 \mathrm{~mm}$ high cylindrical probe. Gel hardness, springiness, cohesiveness, and adhesiveness were observed and recorded directly.

\subsection{Statistics}

Four separate regression equations were obtained of sweet potato starch treated with DS or SG at the following levels: $0,5,15,20,30$, and $50 \%$. The form of the dependent Y-variable and the independent $\mathrm{X}$-variable are as defined in equation $\mathrm{Y}=\mathrm{aX}+\mathrm{b}$, where $\mathrm{Y}$ represents the peak viscosity or setback and $\mathrm{X}$ the level of the added or replaced DS or SG. GLM F-tests for determining peak viscosity or setback equation differences (Y) were based on a full model.

\section{Results and discussion}

\subsection{HPLC}

The HPLC profile of date syrup sugar content revealed two distinct peaks at 8.42 and 8.83 min representing glucose and fructose, respectively. Based on the area under the curves, these results can be interpreted as $55 \mathrm{~g}$ glucose / $100 \mathrm{~g}$ of date syrup sample and $45 \mathrm{~g}$ fructose / $100 \mathrm{~g}$ date syrup sample. Automatically sugar content can be expressed as 55\% glucose and $45 \%$ fructose. These results were not significantly different from what Ahmed \& Ramaswamy (2006) reported, despite the fact that they used specific varieties and a blend of different varieties was used here.

\subsection{Rapid Visco Analyzer measurements (RVA)}

In order to clearly quantify the effect of date syrup (DS) or sugars (SG) on the physical properties of SPS gel and since this study is a replacement and addition experiment, it was necessary to present theoretical profiles of peak viscosity (PV) and setback (SB) of the gels. When calculating the theoretical parameters, we are assuming that the magnitude of PV or SB of plain starch has changed by the same change in starch percent in the event of addition or replacement of the starch with DS or SG. The replacement study was done by simply replacing portion of the starch with DS or SG, whereas in the addition study DS or SG were added to a fixed amount of SPS starch. This is critical, because if we ignore the source of the total solids, the comparison between the effect of DS and SG on SPS gel will be meaningless. Unlike sugars, it is well known that starch contributes directly to the viscosity of the gel. The RVA profile in Figure 1a compares the theoretical value of PV and SB to the actual value obtained directly from RVA. The theoretical value is based on pure starch profile. The RVA profile showed deviation of the replacement or addition profiles from the theoretical. To show how significant this deviation, a multiple regression analysis was applied (Table 1). All equations were statistically significant, as were all slope and interaction coefficients for the models $(\mathrm{p}<0.0001)$, indicating true, non-zero contributions to the prediction equations. SPS exhibited significantly lower PV and SB in both cases of replacement or addition of DS when compared to the theoretical profile, where the actual PV value dropped by 3 folds at 50\% DS replacement. The SB exhibited similar dropped as well. The major drop started at $10 \%$ DS for $\mathrm{PV}$, but SB stared dropping at 25\% DS. This indicates that the reduction in the amount of starch was not the only reason for the drop in PV or SB. Possibly, the effect of DS on the gelatinization temperature and the delay on starch granules swelling are the cause of the lower PV (Eliasson, 1992). Unlike DS, the effect of SG on PV and SB showed increase in PV and SB due to SG addition (Figure $1 \mathrm{c}$ and $\mathrm{d}$ ). This could be attributed to the interaction of SG with the liquid continues phase which gives rise to PV. Comparable to DS, SG addition significantly reduced the $\mathrm{SB}$ relative to the theoretical profile, but the reduction was gradual and intensified at high SG. In both cases of DS and SG, the effect was more evident on the PV than the SB as shown by the slope of the lines representing the actual PV or SB for both DS and SG (Figure 1a-d). Someone may infer from this data, DS can reduce starch retrogradation more than SG which is in agreement with the finding of Mohamed \& Babucurr (2015), but replacement samples exhibited more reduction than addition

Table 1. Multiple regression data for predicting sweet potato starch peak viscosity and setback.

\begin{tabular}{llll}
\hline \multicolumn{1}{c}{ Treatment } & \multicolumn{1}{c}{ Equations } & $\mathrm{R}^{2}$ & P-value \\
\hline Peak Viscosity & & & \\
DS replacement & $\mathrm{Y}=930.35-909.75 \mathrm{X}$ & 0.956 & $<0.0001$ \\
DS addition & $\mathrm{Y}=4895.42+261.64 \mathrm{X}$ & 0.690 & $<0.0001$ \\
SG replacement & $\mathrm{Y}=1357.55-1504.82 \mathrm{X}$ & 0.963 & $<0.0001$ \\
SG addition & $\mathrm{Y}=4805.91+25.933 \mathrm{X}$ & 0.721 & $<0.0001$ \\
& & & \\
Setback & & & \\
DS replacement & $\mathrm{Y}=517.28-384.51 \mathrm{X}$ & 0.780 & $<0.0001$ \\
DS addition & $\mathrm{Y}=1075.87-49.64 \mathrm{X}$ & 0.967 & $<0.0001$ \\
SG replacement & $\mathrm{Y}=547.51-380.83 \mathrm{X}$ & 0.819 & $<0.0001$ \\
SG addition & $\mathrm{Y}=1158.10-5.913 \mathrm{X}$ & 0.689 & $<0.0001$ \\
\hline
\end{tabular}

DS = date syrup; $S G=$ sugars solution . 

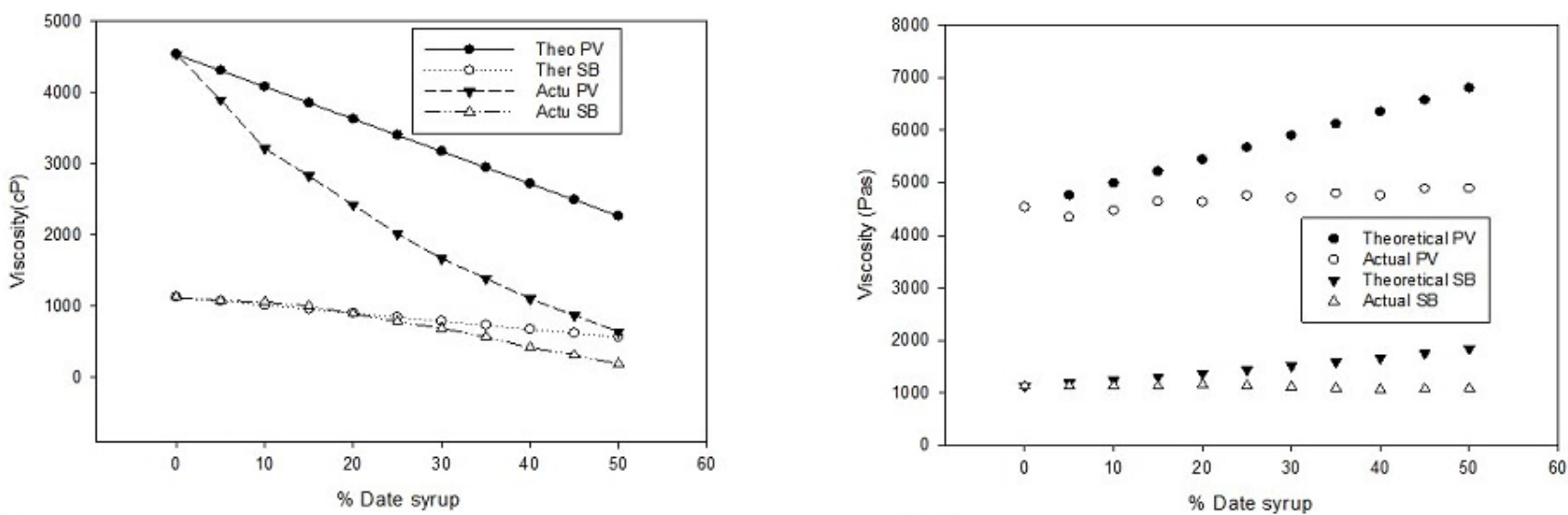

Fig lc.

Fig 1d
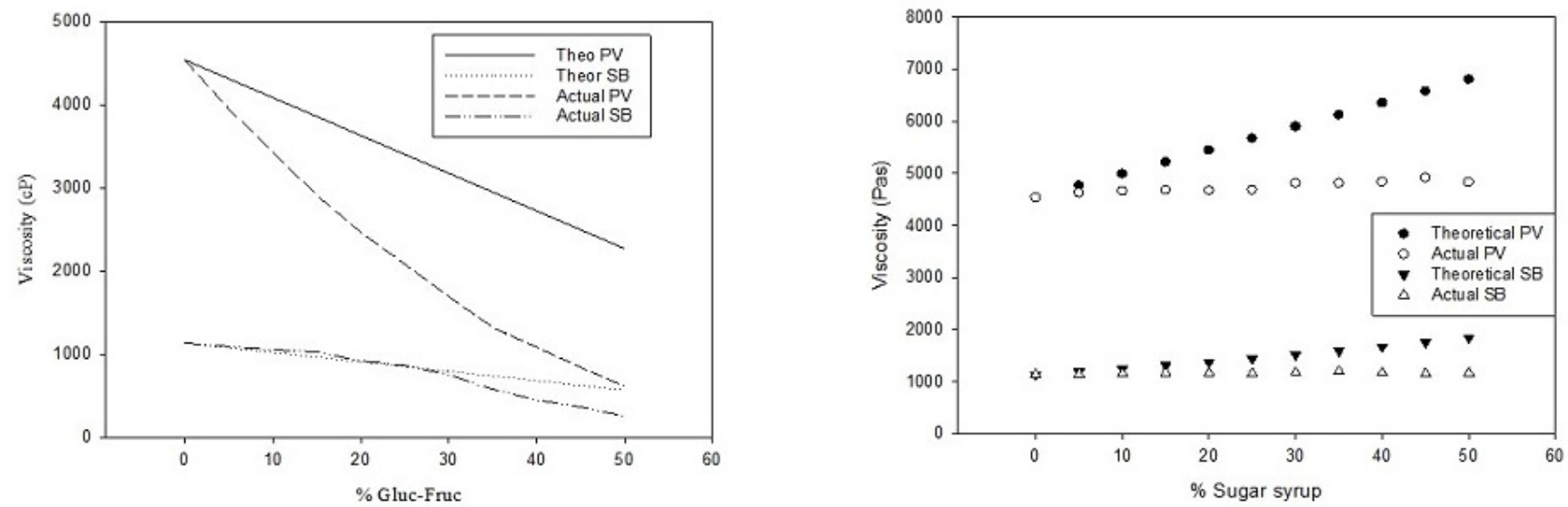

Figure 1. The peak viscosity and setback of date syrup and sweet potato starch: (a). is the replacement with date syrup and (b). is the addition of date syrup; (c). is the replacement with glucose-fructose and (d). is the addition of glucose-fructose.

judging by the slops of the SB lines in Figure 1a and c. Regarding the addition experiment, SB line exhibited plateau at the lowest or the highest DS or SG contents (Figure $1 \mathrm{~b}$ and d). The pasting temperature of the SPS significantly increased by around $15^{\circ} \mathrm{C}$ (data is not shown). This is consistent with the delay in starch granules swelling theory mentioned above. This theory is based on the ability of additives such as sugars to mask starch granules and slow down water penetration or interact with free water molecules and make water less available (Alamri et al., 2016). The data presented in Figure 1a-c and d is reflective of the capacity of DS to have more influence on SPS starch than SG despite the same total solids in either experiments or the similar ratio of glucose to fructose. This date showed that, DS and SG can impact starch pasting properties in a dissimilar way, such as inducing higher peak viscosity, despite the fact that the overall trend of effect may be similar, but the magnitude is different. In view of the slops of the lines, it is apparent that DS or SG had more effect on the PV than SB, whereas DS has more impact on the PV and SB than SG (Figure 1a-d). The capacity of DS to reduce starch retrogradation was reported by Mohamed \& Babucurr (2015).

\subsection{Rheological measurements}

Starch gels were prepared in RVA then loaded on the DHR-Hybrid Rheometer at $25^{\circ} \mathrm{C}$. In order to evidently determine the changes to rheological parameters $\left(G^{\prime}, G^{\prime \prime}, \eta^{*}\right.$ and $\tan \delta$ ), optimum conditions (strain, oscillation frequency, and gap) has to be established.

For the starch system used in this study which is $10 \%$ solids testing at $2 \%$ strain, $0.2 \% \mathrm{~Hz}$ frequency is widely recommended and appeared to meet the requirement for linear viscoelastic region for precooked starch gel (Karim et al., 2000; González-Reyes et al., 2003; Lagarrigue \& Alvarez, 2001; Kasapis et al., 2000). Equally, frequency of oscillation and strain are of enormous importance in the formation or retention of starch gel structure. The $\mathrm{G}^{\prime}$ was used as guide to determine the optimum conditions for this study because it was reported by many researchers to be of more value than G" in describing starch systems and in the same way it was used to determine the linear region of starch containing systems (Hsu et al., 2000; Tattiyakul \& Rao, 2000). 
Neat SPS sample was typical viscoelastic material where G' exceeded $G^{\prime \prime}$ by three folds over all investigated frequencies. The illustrative pattern of the storage modulus $\left(G^{\prime}\right)$ plotted as a function of frequency is indicative of the elastic property of the starch gel (Chang et al., 2014). Figure 2 a-d) represents the $G^{\prime}$ as a function of frequency at four different date syrup (DS) or sugar (SG) levels. Figure 2a and c represent the partial replacement of starch with DS or SG. The figure showed significant drop in $G^{\prime}$ at higher DS or SG in every tested frequency, particularly at $50 \%$ replacement, signifying softer gel. This is consistent with the low PV of the RVA data discussed above, which is in agreement Mohamed \& Babucurr (2015) findings. Stachyose and raffinose were reported to reduce $G^{\prime}$ as well (Zhou et al., 2017).

However, the G' profile of the addition experiment of DS was different, because the $G^{\prime}$ profile exhibited very similar $\mathrm{G}^{\prime}$ as the control (Figure 2b) at all DS levels. Conversely, SG decreased $G^{\prime}$ in the replacement (Figure 2c) and increased it in the addition $(2 \mathrm{~d})$. The increase in $\mathrm{G}^{\prime}$ for the addition study
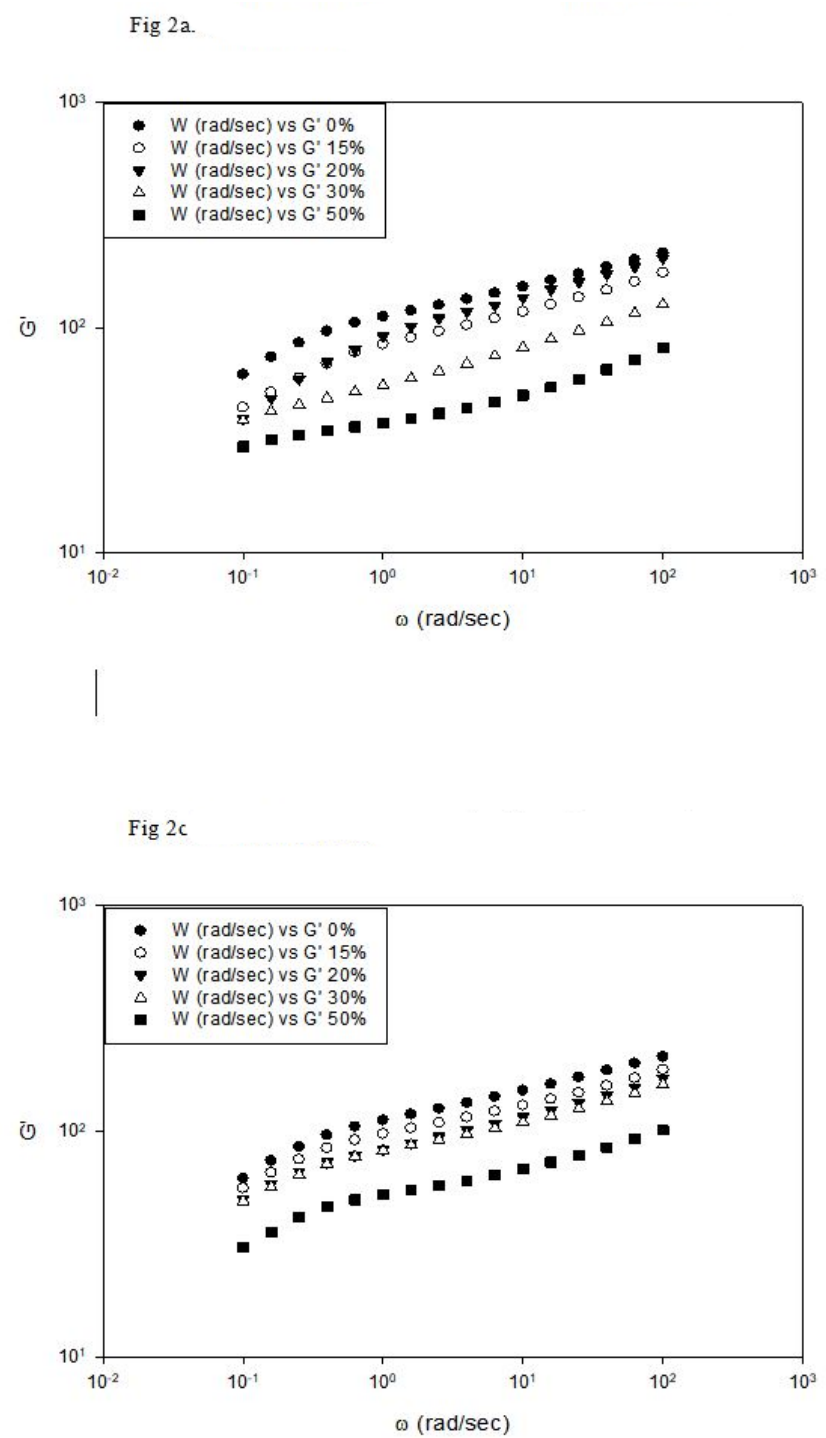

can be attributed to the domination of the solid behavior which was true for SG but not for DS. This demonstrates the different influence of DS on the elastic property of starch gel compared to SG. The influence was also obvious in the replacement experiment where $G^{\prime}$ exhibited gradual drop at higher SG and was less concentration dependent content compared to DS (Fig 2a and c). The increase in $G^{\prime}$ representation for the addition was significant and concentration dependent at higher SG (Figure 2d), therefore, the impact of DS on the G' of SPS gel was not similar to that of SG. The profiles of elastic modulus $\left(G^{\prime}\right)$ as a function of test time, showed significant difference among the replacement/addition experiments and between DS/SG (data is not shown). Exponential increase was observed after 1 ( $\mathrm{rad} / \mathrm{sec})$ frequency but before that $\mathrm{G}^{\prime}$ increase was gradual.

From the above discussion one can conclude that, DS or SG is capable of reducing $G^{\prime}$ of SPS starch gel, but at higher concentrations faster decrease in $G^{\prime}$ was observed. This can be attributed to the increase in amylose content in the continuous
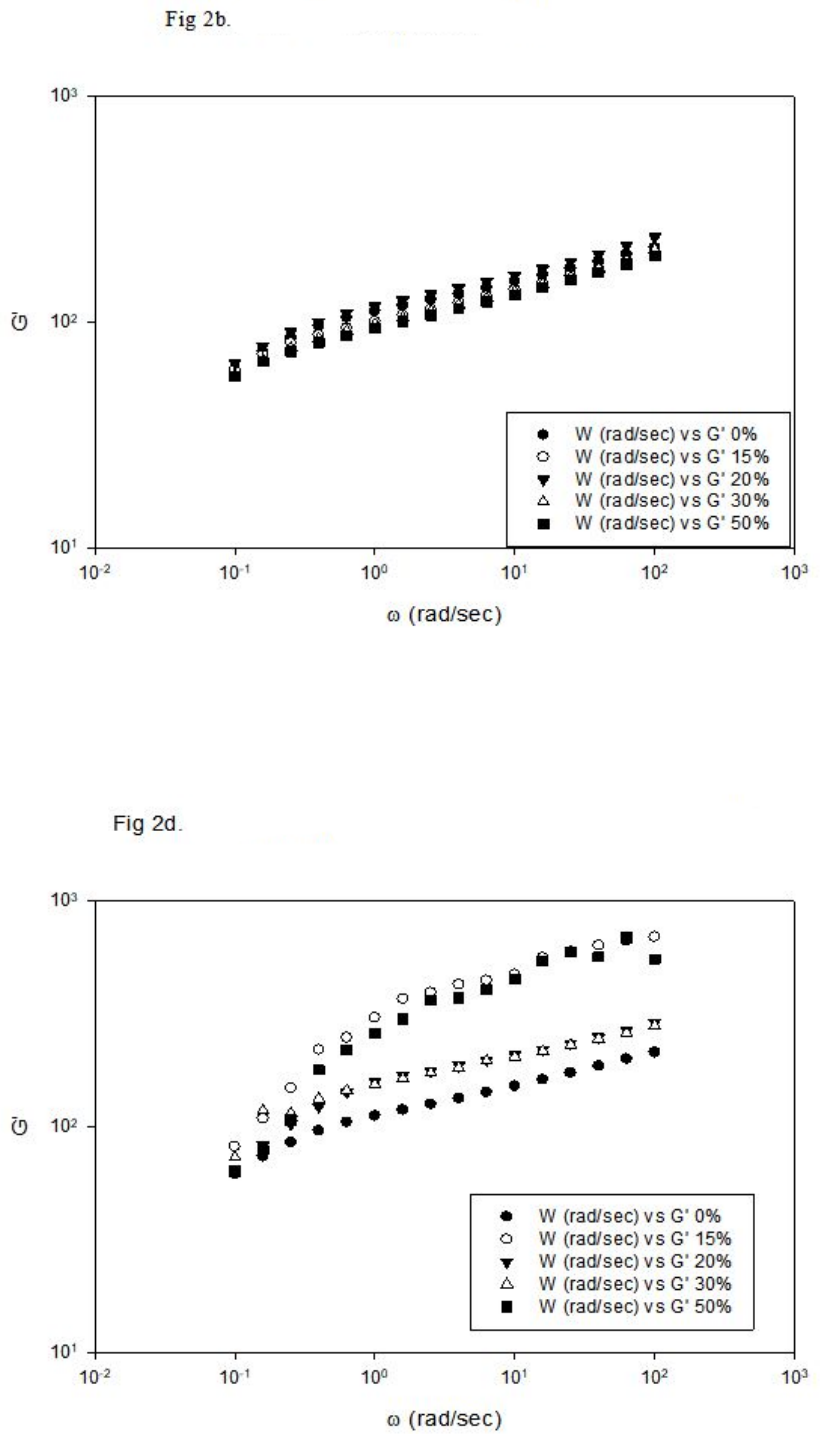

Figure 2. The G' of the sweet potato starch and date syrup or glucose-fructose blends: (a). sweet potato replacement with date syrup; (b). date syrup addition to sweet potato starch; (c). Replacement with glucose-fructose; (d). addition of date syrup. 
phase due to the interaction of sugars with the aqueous phase (Alloncle et al., 1989). This phenomenon indicates thermodynamic incompatibility between starch components (amylose and amylopectin) and DS or SG which commonly leads to phase separation into two coexisting phases; one is less rich in amylose (Dickinson \& McClements 1996). Therefore, amylose network formation is weakened due to the phase separation. Nonetheless, a balance to maintain the gel structure can be reached by lowering the temperature of the precooked system can offset the effect of thermodynamic incompatibility (Bansil, 1993; Tromp et al., 1995).

Complex shear modulus is a measure used to determine elastic susceptibility of elastic materials depending on the induced stress on the material at a specific frequency (Mason \& Weitz, 1995). Since starch gels are viscoelastic material capable of storing or dissipating energy depending on the applied frequency, the steep increase in $G^{\prime}$ occurred at lower frequencies. That explains why steep increase at lower frequency occurred in this study as indicate by the SG addition (Figure $2 \mathrm{~d}$ ). This could be due to the interaction of SPS and the sugars or by the high affinity of sugars for water and the outcome is a more elastic gel (Choi \& Chang, 2012). In general, factors that affect starch gel structural development have direct effect of either limited amylose leaching out of the granule or interaction with the leached amylose or both. Faster $G^{\prime}$ increase can also be attributed to the increase in amylose content in the continuous phase due to the presence of the sugars (Alloncle et al., 1989). No specific order was observed with respect to increase in $\mathrm{G}^{\prime}$ as a function of SG addition, where $15 \%$ and $50 \%$ SG of the addition experiment appears to have similar effect on $G^{\prime}$ profile (Figure $2 \mathrm{~d}$ ) and significantly higher than the $20 \%$ and $30 \%$ SG.

In this situation, the effect of DS seems to be the opposite of SG because all concentrations appear to have similar effect on $G^{\prime}$ at all tested frequencies (Figure $2 b$ ). Nonetheless, the addition of sugars is known to increase starch gelatinization temperature and suppressing the peak viscosity causing limited starch gelatinization as shown by the RVA data discussion above. This phenomenon was observed at the beginning of starch gelatinization, but once the gelatinization started it progresses in a way identical to that in pure water. Besides, the gelatinization temperature is dependent on the molecular weight or the concentration of the solute, which is directly related to granules swelling, the main precursor to full gelatinization. Although $G^{\prime}$ pattern of starch system containing gums was shown to increase at the beginning, after that it start moving to plateau (Choi \& Yoo, 2009)

\section{4 $\log G^{\prime}$ and $G^{\prime \prime}$ versus Frequency}

Starch gels can be considered true gel-like because it exhibits much higher $G^{\prime}$ than $G^{\prime \prime}$ (Chang et al., 2014). Therefore, the linear regression of the $\log$ of $G^{\prime}$ or $G^{\prime \prime}$ 'versus log of frequency as shown in equations 1 and 2 , allowed the calculation of $n$ ' and $\mathrm{n}$ " ' or $\mathrm{k}$ ' or $\mathrm{k}$ " where $\mathrm{n}$ is the slope of line and $\mathrm{k}$ is the intercept

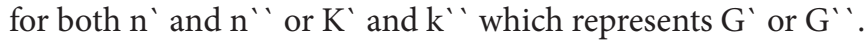

$G^{\prime}=k^{\prime} \omega^{n}$

$\log G^{\prime}=\log k^{\prime}+n^{\prime} \log \omega$

Where, $\mathrm{n}$ ' represents the shear behavior index and $\mathrm{k}$ ' is the consistency index of the power law. From structural stand point, the slope for true gels should be zero and weak gels must exhibit positive slope (Choi \& Chang, 2012), whereas K' is higher than $\mathrm{K}^{\prime}$ ' with frequency dependency. Overall, the $\mathrm{k}$ ' for samples with DS or SG at all concentrations was much higher than $\mathrm{k}^{\prime}$ '. For example, $\mathrm{K}^{\prime}$ of DS-containing SPS samples ranged from 3.65-4.39, whereas K' exhibited 1.83-2.82 (Table 2). With some exceptions, higher DS or SG further reduced the k value (Table 2) indicating reduction in the elastic property of SPS gels. Such reduction can simply be related to the disruption of the amylose network which is the main architect of the gel formation. The slope of DS or SG concentration versus K' was used to compare the effect of DS on the elastic property of SPS compared to SG (Table 2). The K' of DS-containing replacement samples exhibited -0.024 whereas for SG it was -0.016 , but for the addition of DS samples showed no specific pattern and SG had slope of 0.013. It is clear how different the impact of

Table 2. Effect of date syrup and sugars (glucose+fructose) on the n ', k', n ' , and k ' representing G' and G' ' of sweet potato starch, respectively, according to equation $2 ; \log \mathrm{G}^{\prime}=\log \mathrm{k}^{\prime}+\mathrm{n}^{`} \log \omega$.

\begin{tabular}{|c|c|c|c|c|c|c|}
\hline \multirow{2}{*}{$\mathrm{DS}^{1}$ or $\mathrm{SG}^{2}$} & \multicolumn{3}{|c|}{$\mathrm{G}^{\prime}$} & \multicolumn{3}{|c|}{$\mathrm{G}^{\prime \prime}$} \\
\hline & $\mathrm{n}^{\prime}$ & $\mathrm{K}^{\prime}$ (Pas) & $\mathrm{R}^{2}$ & $\mathrm{n}^{\prime \prime}$ & $\mathrm{K}^{\prime \prime}(\mathrm{Pas})$ & $\mathrm{R}^{2}$ \\
\hline $0 \%$ (control) & 0.16 & 4.64 & 0.98 & 0.24 & 2.95 & 0.99 \\
\hline $20 \%$ DS Re & 0.21 & 4.39 & 0.96 & 0.28 & 2.82 & 0.98 \\
\hline $30 \%$ DS Re & 0.17 & 4.02 & 0.99 & 0.25 & 2.59 & 0.97 \\
\hline $50 \%$ DS Re & 0.14 & 3.65 & 0.97 & 0.33 & 1.83 & 0.95 \\
\hline $20 \%$ DS Ad & 0.17 & 4.21 & 0.98 & 0.23 & 2.76 & 0.99 \\
\hline $30 \%$ DS Ad & 0.17 & 1.04 & 0.97 & 0.23 & 2.68 & 0.98 \\
\hline $50 \%$ DS Ad & 0.16 & 4.01 & 0.99 & 0.24 & 2.70 & 0.99 \\
\hline $20 \%$ SG Re & 0.16 & 3.91 & 0.99 & 0.24 & 2.48 & 0.64 \\
\hline $30 \%$ SG Re & 0.16 & 3.87 & 0.98 & 0.24 & 2.29 & 0.95 \\
\hline $50 \%$ SG Re & 0.15 & 3.46 & 0.97 & 0.29 & 1.80 & 0.98 \\
\hline $20 \%$ SG Ad & 0.18 & 4.36 & 0.91 & 0.24 & 2.68 & 0.91 \\
\hline $30 \%$ SG Ad & 0.15 & 4.42 & 0.92 & 0.21 & 284 & 0.95 \\
\hline $50 \%$ SG Ad & 0.32 & 4.73 & 0.88 & 0.41 & 2.38 & 0.88 \\
\hline
\end{tabular}

DS = Date Syrup; $S G=$ glucose $/$ fructose. 
DS on the elastic property of SPS as indicated by the variation between the slopes.

Nevertheless, in present study the flow behavior index (n) was less than 1.0 indicating pseudoplastic gels with shear thinning behavior. These results are in agreement with the work of Zhou et al. (2017) and Lee \& Yoo (2014). In contrast to the data presented here, Choi \& Chang (2012) reported increase in the consistency index $(\mathrm{K})$ value for buckwheat starch-galactomannan blends. As it was mentioned earlier, the molecular weight of the solute is one of the determining factors on starch gels rheological properties, consequently, gums have a tendency to increase the $\mathrm{K}^{\prime}$ and $\mathrm{K}^{\prime}$ '.

The profile of the frequency-dependent complex viscosity is illustrated in Figure 3. Sweet potato starch gels blend with DS exhibited shear thinning similar to the profile of the control (Figure 3a). This outcome is expected because complex viscosity is a product of the square root of $\left(G^{\prime}\right)^{2}+\left(G^{\prime \prime}\right)^{2}$ divided by the oscillation frequency and $G^{\prime}$ of DS addition profile was very similar to the control (Figure 2b). Conversely, higher complex viscosity was observed for blends containing SG. Once more, SG addition interfered with the physical properties of SPS gels more than DS as seen in the example of $G^{\prime}$ illustrated in Figure $2 \mathrm{~b}$ and $\mathrm{d}$. Significant difference between the complex viscosities of the blends was observed with respect to the SG concentration, where $15 \%$ and $50 \%$ SG had similar effect versus $20 \%$ and $30 \%$ (Figure $3 \mathrm{~b}$ ). This could be attributed to the concentration of SG in the continuous phase. As per other researchers, the reason for the solid-like behavior of starches in the presence of xanthan gum and other gums was due to the reinforcement of the network of the starch gel (Mandala \& Bayas, 2004; Ross-Murphy, 1984). These reports point out the major difference between gums and sugars with respect to their influence on starch gels rheological behavior.

Two mechanisms were proposed by researchers on the synergetic effect of gums and other lower-molecular weight carbohydrates on starch gels. One mechanism accredited the effect to the interaction between starch exudate and the solute which leads to increase of the gum in the continuous phase. The other mechanism was the effect of gums on the shape and the size of the starch granule as well as the granule to granule interaction. Although DS or SG reduced the $G^{\prime}$, at higher concentrations faster decrease in $G^{\prime}$ was observed. This can be attributed to amylose content increase in the continuous phase due to the interaction of sugars with the aqueous phase (Alloncle \& Doublier, 1991). This phenomena indicates thermodynamic incompatibility between starch components (amylose and amylopectin) and DS or SG which commonly leads to phase separation into two coexisting phases, one is less rich in amylose (Dickinson \& McClements, 1996; Tolstoguzov, 1986). Therefore, amylose network formation is weakened due to the phase separation. Nonetheless, a balance between maintaining the gel structure can be reached by lowering the temperature of the system which can offset the effect of thermodynamic incompatibility (Bansil, 1993; Tromp et al., 1995).

\section{$3.5 \tan \delta$}

The $\tan \delta$ of all samples was in the range of $0.02-0.20$ indicating elastic behavior since it is less than one. In the range of 20 to $60(\mathrm{rad} / \mathrm{sec})$ frequencies, $\tan \delta$ of SPS exhibited concentration dependency where the $50 \%$ replacement or addition showed higher $\tan \delta$ than the control (Figure $4 \mathrm{a}$ and $\mathrm{b}$ ). This means that in the incident of lower $\tan \delta$ (high $\mathrm{G}^{\prime}$ ), starch gel was more elastic compared to those with higher $\tan \delta$ (Lower $G^{\prime}$ ). Samples with higher $\tan \delta$ point to less structured and more viscous gel. Steep increase in $\tan \delta$ was observed at frequencies lower than $20(\mathrm{rad} / \mathrm{sec})$ and after that the profile started taking plateau representation (Figure $4 \mathrm{a}$ and $\mathrm{b}$ ). In contrast, gums have a propensity for increasing $\tan \delta$ but maintained plateau profile regardless of frequency adjustment (Chun \& Yoo, 2006). The $\tan \delta$ plateau indicates unchanged solid like behavior of the gel. Therefore, at low frequency gel network formation process is
Fig 3a.

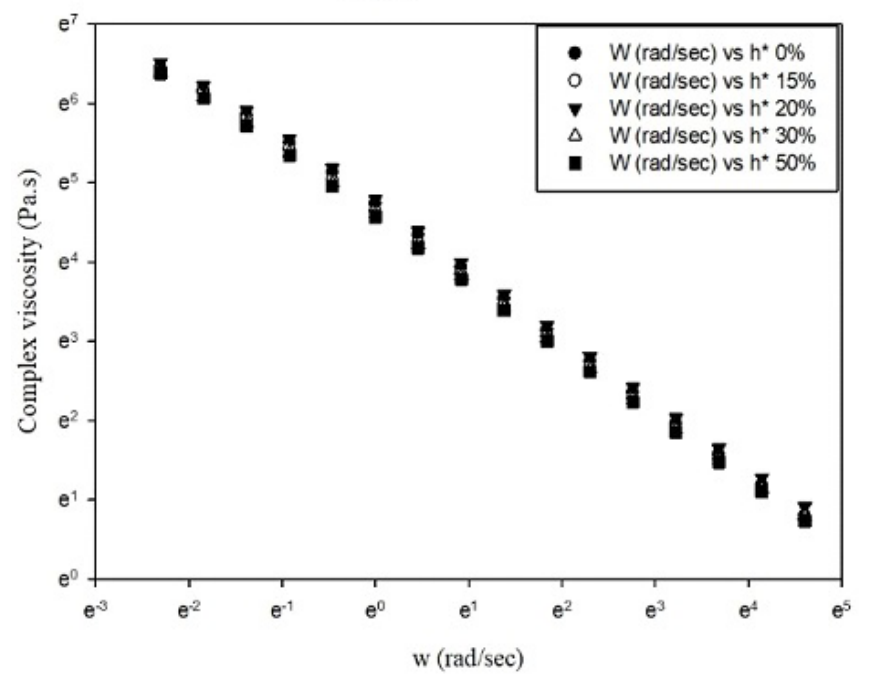

Fig 3b.

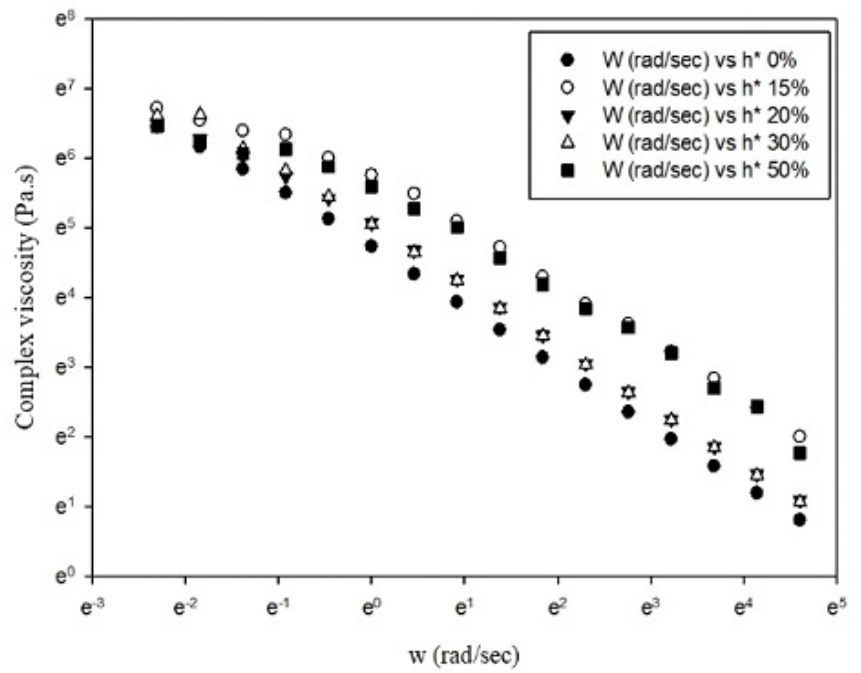

Figure 3. The complex viscosity of the sweet potato and date syrup or glucose-fructose blends: (a). complex viscosity of the addition of date syrup to sweet potato starch. (b). complex viscosity of the addition of glucose-fructose to sweet potato starch. 
accelerating and gradually the solid-like behavior is dominating then became unchanged when tan reached plateau (Figure 4).

Unlike gums and high molecular weight carbohydrates, the effect of sugars on starch physicochemical properties was explained by a number of researchers to be caused by penetration of small sugars into the amorphous regions of starch granules
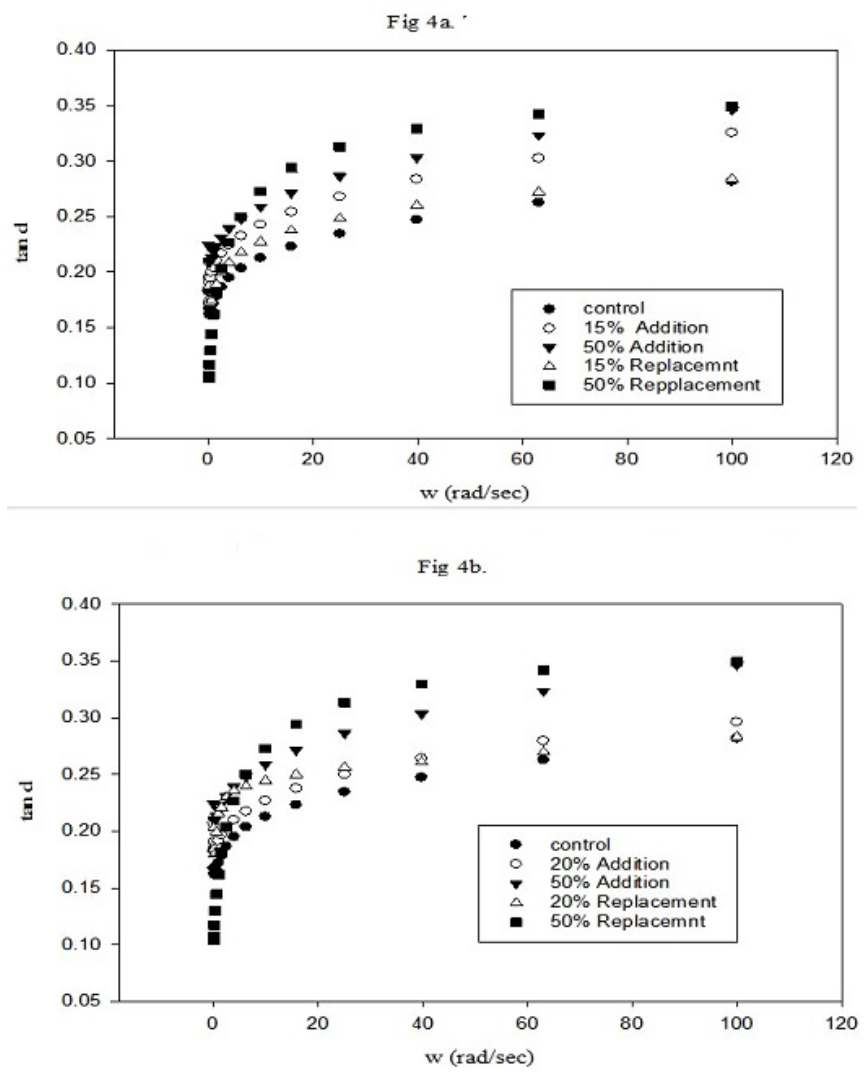

Figure 4. The $\tan \delta$ of the addition and replacement of date syrup and glucose-fructose to sweet potato starch. 4 a. the addition and replacement of date syrup at 0,15 , and $50 \% .4 \mathrm{~b}$. The addition and replacement of glucose-fructose at 0,15 , and $50 \%$. instigating complex formation between sugars and amylose. This caused delay on starch swelling, amylose leaching, and overall limited starch gelatinization. Other investigators reported that the effect is attributable to concentration of sugars in the continuous phase of the system (Sikora \& Pielichowski, 1999; Chiotelli et al., 2000). Regarding the rate of amylose network formation, the addition of sugars caused large decrease in the rate of preliminary conformational organization of amylose network (retrogradation) during cooling, which is corroborated by the decrease in the G' (Evageliou et al., 2000). Either of the theories can be used to explain the change in $G^{\prime}$ of the starch due to DS or SG as well as the peak viscosity of the RVA test. It is recognized that minor components of date syrup could have contributed to the different effect of DS on SPS compared to SG but the effect of the major components (glucose and fructose) will assist in predicting starch-DS system application in processing such as extrusion.

\subsection{Gel texture}

The gel hardness of the replaced starch with DS or SG was lower at higher replacement level (Table 3) indicates weak gel network primarily due to the absence of amylose, which is consistent with values obtained for the $G^{\prime}$. As expected, samples with addition of DS or SG exhibited higher gel hardness because of the increase in solid content. This data is in agreement with RVA data despite the difference in testing temperature, where RVA test was done at $50^{\circ} \mathrm{C}$ and gel hardness at room temperature. This can be attributed to the faster amylose retrogradation in the presence of the DS and SG (addition) due to the interaction of the solute with the water allowing amylose molecules to retrograde. In addition to the shortage of amylose due to replacement, it appears that solute interacted with amylose and partially prevented retrogradation producing softer gel. The slope of decrease in hardness for DS or SG replacement was pretty close -2.72 and -2.84 for the DS compared to SG, respectively, but the addition experiment exhibited 1.87 and -2.52 for DS and SG, respectively.

Table 3. Sweet potato starch gel textural characteristics.

\begin{tabular}{|c|c|c|c|c|c|c|}
\hline$\% \mathrm{DS}^{1}$ or $\mathrm{SG}^{2}$ & \multicolumn{2}{|c|}{ Hardness (g) } & \multicolumn{2}{|c|}{ Cohesiveness } & \multicolumn{2}{|c|}{ Adhesiveness (mJ) } \\
\hline \multicolumn{7}{|c|}{ Replacement } \\
\hline & DS & SG & DS & SG & DS & SG \\
\hline 0 & $146 \pm 2.6 \mathrm{a}$ & $146 \pm 1.2 \mathrm{a}$ & $0.41 \pm 0.01 \mathrm{~b}$ & $0.41 \pm 0.02 \mathrm{~b}$ & $0.40 \pm 0.01 \mathrm{~b}$ & $0.40 \pm 0.05 c$ \\
\hline 15 & $120 \pm 6.2 b$ & $127 \pm 7.2 b$ & $0.44 \pm 0.03 b$ & $0.42 \pm 0.03 \mathrm{~b}$ & $1.00 \pm 0.03 \mathrm{a}$ & $0.40 \pm 0.01 \mathrm{c}$ \\
\hline 20 & $105 \pm 4.0 \mathrm{c}$ & $116 \pm 6.4 \mathrm{c}$ & $0.41 \pm 0.02 b$ & $0.37 \pm 0.02 c$ & $0.90 \pm 0.04 \mathrm{a}$ & $0.90 \pm 0.03 \mathrm{a}$ \\
\hline 30 & $76 \pm 3.50 \mathrm{~d}$ & $63 \pm 8.00 \mathrm{~d}$ & $0.35 \pm 0.02 c$ & $0.42 \pm 0.01 \mathrm{~b}$ & $0.70 \pm 0.02 \mathrm{a}$ & $0.70 \pm 0.02 b$ \\
\hline 50 & $13 \pm 1.20 \mathrm{e}$ & $13 \pm 2.30 \mathrm{e}$ & $0.71 \pm 0.04 \mathrm{a}$ & $0.57 \pm 0.03 \mathrm{a}$ & $0.20 \pm 0.03 \mathrm{~b}$ & $0.10 \pm 0.02 \mathrm{a}$ \\
\hline \multicolumn{7}{|c|}{ Addition } \\
\hline & DS & SG & DS & SG & DS & SG \\
\hline 0 & $146 \pm 2.6 \mathrm{~d}$ & $146 \pm 1.2 \mathrm{c}$ & $0.41 \pm 0.01 \mathrm{~b}$ & $0.41 \pm 0.02 \mathrm{c}$ & $0.40 \pm 0.01 \mathrm{c}$ & $0.40 \pm 0.5 c$ \\
\hline 15 & $145 \pm 2.5 \mathrm{~d}$ & $165 \pm 0.9 \mathrm{a}$ & $0.47 \pm 0.03 a$ & $0.38 \pm 0.01 \mathrm{c}$ & $0.30 \pm 0.04 \mathrm{~d}$ & $1.50 \pm 0.5 \mathrm{a}$ \\
\hline 20 & $156 \pm 5.1 \mathrm{bc}$ & $148 \pm 6.2 \mathrm{c}$ & $0.52 \pm 0.01 \mathrm{a}$ & $0.51 \pm 0.03 \mathrm{a}$ & $2.00 \pm 0.03 \mathrm{a}$ & $0.70 \pm 0.4 \mathrm{~b}$ \\
\hline 30 & $161 \pm 4.6 b$ & $105 \pm 5.7 \mathrm{~d}$ & $0.45 \pm 0.04 \mathrm{ab}$ & $0.48 \pm 0.04 \mathrm{~b}$ & $0.90 \pm 0.01 \mathrm{~b}$ & $0.80 \pm 0.5 b$ \\
\hline 50 & $212 \pm 8.1 \mathrm{a}$ & $76 \pm 4.00 \mathrm{e}$ & $0.46 \pm 0.02 \mathrm{a}$ & $0.50 \pm 0.01 \mathrm{a}$ & $0.40 \pm 0.04 \mathrm{c}$ & $0.50 \pm 0.4 \mathrm{c}$ \\
\hline
\end{tabular}

DS=date syrup; SG = sugar solutions; means within same column with similar letters are not Significantly different ( $p>0.05)$. 
Unlike hardness, cohesiveness was also greatly dropped as a function of DS or SG content because this parameter is a product of hardness, except for SG replacement (Table 3). The gel cohesiveness exhibited contrasting data for DS or SG replacement where the slope of increase was 5.39 and 3.21 for DS and SG, respectively, but the addition samples exhibited 0.001 and 0.002 slopes for the DS and SG, respectively. Despite the different slopes, cohesiveness was not significantly affected by DS and SG in the addition data (Table 3), but Ds exhibited better data order than SG. Adhesiveness data showed no specific pattern for both DS and SG. Largely, the flowing properties of starch gels are dependent on several factors such as the rheological features of the formed amylose network, the volume fraction and the rigidity of the swollen starch granules, in addition to the interactions between dispersed and continuous phases of the gel. These factors are dependent on the amylose content and the structure of the amylopectin in terms of degree of branching (Biliaderis, 1998).

\section{Conclusion}

The rheological parameters of sweet potato starch-date syrup and glucose/fructose sugar blends gels were tested. The data presented here verified the dissimilar effect of DS compared to SG on the flowing properties of sweet potato starch gel. Specifically, the pasting properties, dynamic rheological parameters, and gel textural properties were impacted by DS and SG. The G' and G" were decreased as the date syrup and sugar blends increased signifying weak-gel behavior. The increase in $\tan \delta$ at lower frequencies indicates that the presence of DS and SG lowered the elastic property of the starch gel, while at higher frequencies $\mathrm{G}^{\prime}$ tends to plateau. Some of these impacts are demonstrated to take place during preliminary gel conformational organization of amylose network during cooling and others after gels are formed subsequent cooling. The magnitude of DS influence compared to SG was indicated by the data presented here. It is acknowledged that minor components of date syrup, which are not considered in this study, could have contributed to the change in rheological parameters presented here, but the effect of major components of DS (sugars) can assist in predicting changes to starch-date syrup system during various processing applications such as extrusion.

\section{Acknowledgements}

The authors extend their appreciation to the Deanship of Scientific Research at King Saud University for funding this work through research group no RGP-114

\section{References}

Ahmad, F. B., \& Williams, P. A. (1999). Effect of sugars on the thermal and rheological properties of sago starch. Biopolymers: Original Research on Biomolecules, 50(4), 401-412. http://dx.doi.org/10.1002/ (SICI) 1097-0282(19991005)50:4<401::AID-BIP6>3.0.CO;2-V. PMid:10423549.

Ahmed, J., \& Ramaswamy, H. S. (2006). Physico-chemical properties of commercial date pastes (Phoenix dactylifera). Journal of Food Engineering, 76(3), 348-352. http://dx.doi.org/10.1016/j. jfoodeng.2005.05.033.
Alamri, M., Hussain, S., Mohamed, A., Abdo Qasem, A. A., \& Ibraheem, M. (2016). A study on the effect of black cumin extract on the swelling power, textural, and pasting properties of different starches. Stärke, 68(11-12), 1233-1243. http://dx.doi.org/10.1002/star.201500330.

Al-Hooti, S. N., Sidhu, J. S., Al-Saqer, J. M., \& Al-Othman, A. (2002). Chemical composition and quality of date syrup as affected by pectinase/cellulase enzyme treatment. Food Chemistry, 79(2), 215220. http://dx.doi.org/10.1016/S0308-8146(02)00134-6.

Al-Khateeb, A. A. (2008). Enhancing the growth of date palm (Phoenix Dactylifera) in vitro tissue by adding date syrup to the culture medium. Scientific Journal of King Faisal University, 9(1), 71-85. [Basic and Applied Sciences]

Alloncle, M., \& Doublier, J. L. (1991). Viscoelastic properties of maize starch/hydrocolloid pastes and gels. Food Hydrocolloids, 5(5), 455467. http://dx.doi.org/10.1016/S0268-005X(09)80104-5.

Alloncle, M., Lefebvre, J., Llamas, G., \& Doublier, J. L. (1989). A rheological characterization of cereal starch-galactomannan mixtures. Cereal Chemistry, 66(2), 90-93.

Association of Official Analytical Chemists - AOAC. (1990). Official Methods (16.12) of Analysis of AOAC International (16th ed.). AOAC: Washington.

Baek, M. H., Yoo, B., \& Lim, S. T. (2004). Effects of sugars and sugar alcohols on thermal transition and cold stability of corn starch gel. Food Hydrocolloids, 18(1), 133-142. http://dx.doi.org/10.1016/ S0268-005X(03)00058-4.

Bansil, R. (1993). Phase separation in polymer solutions and gels. Journal de Physique. IV, 3(C1), C1-C225. http://dx.doi.org/10.1051/ jp4:1993119.

Biliaderis, C. G. (1998). Structures and phase transitions of starch polymers. In R. H. Walker (Ed.), Polysaccharide association structures in food (pp. 57-168). New York: Marcel Dekker, Inc.

Chang, Y. H., Lim, S. T., \& Yoo, B. (2014). Dynamic rheology of corn starch-sugar composites. Journal of Food Engineering, 64(4), 521527. http://dx.doi.org/10.1016/j.jfoodeng.2003.08.017.

Chiotelli, E., Rolée, A., \& Le Meste, M. (2000). Effect of sucrose on the thermomechanical behavior of concentrated wheat and waxy corn starch-water preparations. Journal of Agricultural and Food Chemistry, 48(4), 1327-1339. http://dx.doi.org/10.1021/jf990817f. PMid:10775393.

Choi, D. W., \& Chang, Y. H. (2012). Steady and dynamic shear rheological properties of buckwheat starch-galactomannan mixtures. Preventive Nutrition and Food Science, 17(3), 192-196. http://dx.doi.org/10.3746/ pnf.2012.17.3.192. PMid:24471083.

Choi, H. M., \& Yoo, B. (2009). Steady and dynamic shear rheology of sweet potato starch-xanthan gum mixtures. Food Chemistry, 116(3), 638-643. http://dx.doi.org/10.1016/j.foodchem.2009.02.076.

Chun, S. Y., \& Yoo, B. (2006). Steady and dynamic shear rheological properties of sweet potato flour dispersions. European Food Research and Technology, 223(3), 313-319. http://dx.doi.org/10.1007/s00217005-0205-9.

Dickinson, E., \& McClements, D. J. (1996). Advances in food colloids (pp. 81-101). Glasgow: Blackie/Chapman \& Hall. http://dx.doi. org/10.1007/978-1-4613-1223-9_3.

Eliasson, A. C. (1992). A calorimetric investigation of the influence of sucrose on the gelatinization of starch. Carbohydrate Polymers, 18(2), 131-138. http://dx.doi.org/10.1016/0144-8617(92)90135-D.

Evageliou, V., Richardson, R. K., \& Morris, E. R. (2000). Effect of sucrose, glucose and fructose on gelation of oxidised starch. Carbohydrate Polymers, 42(3), 261-272. http://dx.doi.org/10.1016/ S0144-8617(99)00158-7. 
González-Reyes, E., Méndez-Montealvo, G., Solorza-Feria, J., ToroVazquez, J. F., \& Bello-Perez, L. A. (2003). Rheological and thermal characterization of Okenia hypogaea (Schlech. \& Cham.) starch. Carbohydrate Polymers, 52(3), 297-310. http://dx.doi.org/10.1016/ S0144-8617(02)00291-6.

Gunaratne, A., Ranaweera, S., \& Corke, H. (2007). Thermal, pasting, and gelling properties of wheat and potato starches in the presence of sucrose, glucose, glycerol, and hydroxypropyl $\beta$-cyclodextrin. Carbohydrate Polymers, 70(1), 112-122. http://dx.doi.org/10.1016/j. carbpol.2007.03.011.

Hsu, S., Lu, S., \& Huang, C. (2000). Viscoelastic changes of rice starch suspensions during gelatinization. Journal of Food Science, 65(2), 215-220. http://dx.doi.org/10.1111/j.1365-2621.2000.tb15982.x.

Karim, A. A., Norziah, M. H., \& Seow, C. C. (2000). Methods for the study of starch retrogradation. Food Chemistry, 71(1), 9-36. http:// dx.doi.org/10.1016/S0308-8146(00)00130-8.

Kasapis, S., Sablani, S. S., \& Biliaderis, C. G. (2000). Dynamic oscillation measurements of starch networks at temperatures above $100 \mathrm{C}$. Carbohydrate Research, 329(1), 179-187. http://dx.doi.org/10.1016/ S0008-6215(00)00161-0. PMid:11086697.

Kim, A. S. (1992). Effects of sugars and emulsifiers on starch gelatinization evaluated by differential scanning calorimetry. Cereal Chemistry, 69, 212-217.

Lagarrigue, S., \& Alvarez, G. (2001). The rheology of starch dispersions at high temperatures and high shear rates: a review. Journal of Food Engineering, 50(4), 189-202. http://dx.doi.org/10.1016/S02608774(00)00239-9.

Lee, S., \& Yoo, B. (2014). Effect of sucrose addition on rheological and thermal properties of rice starch-gum mixtures. International Journal of Food Engineering, 10(4), 849-856. http://dx.doi.org/10.1515/ ijfe-2014-0072.

Ma'abreh, A. S., Abu-Salah, K. M., Al-Awaadh, A. M. \& Mohamed, A. A. (2018). Properties of gel formulated from nanoparticles of palm date syrup. Journal of Food Process Engineering, 41(3), e12890. http:// dx.doi.org/10.1111/jfpe.12890.

Maaruf, A. G., Man, Y. C., Asbi, B. A., Junainah, A. H., \& Kennedy, J. F. (2001). Effect of water content on the gelatinisation temperature of sago starch. Carbohydrate Polymers, 46(4), 331-337. http://dx.doi. org/10.1016/S0144-8617(00)00335-0.

Mandala, I. G., \& Bayas, E. (2004). Xanthan effect on swelling, solubility and viscosity of wheat starch dispersions. Food Hydrocolloids, 18(2), 191-201. http://dx.doi.org/10.1016/S0268-005X(03)00064-X.

Mason, T. G., \& Weitz, D. A. (1995). Optical measurements of frequency-dependent linear viscoelastic moduli of complex fluids. Physical Review Letters, 74(7), 1250-1253. http://dx.doi.org/10.1103/ PhysRevLett.74.1250. PMid:10058972.

Miles, M. J., Morris, V. J., Orford, P. D., \& Ring, S. G. (1985). The roles of amylose and amylopectin in the gelation and retrogradation of starch. Carbohydrate Research, 135(2), 271-281. http://dx.doi. org/10.1016/S0008-6215(00)90778-X.

Mohamed, I. O., \& Babucurr, J. (2015). Effect of date syrup on pasting, rheological, and retrogradation properties of corn starch gels. Stärke, 67(7-8), 709-715. http://dx.doi.org/10.1002/star.201500062.

Mohamed, I. O., \& Babucurr, J. (2017). Pasting, rheological, and retrogradation properties of low-amylose rice starch with date syrup. Food Science \& Technology International, 23(6), 550-558. http://dx.doi.org/10.1177/1082013217711344. PMid:28549408.

Noda, T., Takahata, Y., Sato, T., Kumagai, T., \& Yamakawa, O. (1998). Starch properties and cell-wall material contents in sweet potatoes as affected by flesh color, cultivation method and year. Journal of Applied Glycoscience, 45(1), 1-9.

Pongsawatmanit, R., Temsiripong, T., \& Suwonsichon, T. (2007). Thermal and rheological properties of tapioca starch and xyloglucan mixtures in the presence of sucrose. Food Research International, 40(2), 239-248. http://dx.doi.org/10.1016/j.foodres.2006.10.013.

Ross-Murphy, S. B. (1984) Rheological methods. In H. W. S. Chan (Ed.), Biophysical methods in food research (pp. 138-199). London. Blackwell Scientific Publications.

Sikora, M., \& Pielichowski, K. (1999). Rheological properties of some starch-water-sugar systems. International Journal of Food Science \& Technology, 34(4), 371-383. http://dx.doi.org/10.1046/j.13652621.1999.00283.x.

Sopade, P. A., Halley, P. J., \& Junming, L. L. (2004). Gelatinisation of starch in mixtures of sugars. II. Application of differential scanning calorimetry. Carbohydrate Polymers, 58(3), 311-321. http://dx.doi. org/10.1016/j.carbpol.2004.07.007.

Tattiyakul, J., \& Rao, M. A. (2000). Rheological behavior of crosslinked waxy maize starch dispersions during and after heating. Carbohydrate Polymers, 43(3), 215-222. http://dx.doi.org/10.1016/ S0144-8617(00)00160-0.

Tolstoguzov, V. B. (1986). Functional properties of protein-polysaccharide mixtures. In J. R. Mitchell \& D. A. Ledward (Eds.), Functional properties of food macromolecules (pp. 385-415). London: Elsevier Applied Science.

Tromp, R. H., Rennie, A. R., \& Jones, R. A. L. (1995). Kinetics of the simultaneous phase separation and gelation in solutions of dextran and gelatin. Macromolecules, 28(12), 4129-4138. http://dx.doi. org/10.1021/ma00116a012.

Zhang, X., Tong, Q., Zhu, W., \& Ren, F. (2013). Pasting, rheological properties and gelatinization kinetics of tapioca starch with sucrose or glucose. Journal of Food Engineering, 114(2), 255-261. http:// dx.doi.org/10.1016/j.jfoodeng.2012.08.002.

Zhou, D. N., Zhang, B., Chen, B., \& Chen, H. Q. (2017). Effects of oligosaccharides on pasting, thermal and rheological properties of sweet potato starch. Food Chemistry, 230, 516-523. http://dx.doi. org/10.1016/j.foodchem.2017.03.088. PMid:28407943. 